Archives de sciences sociales des religions

111 | juillet-septembre 2000

Varia

\title{
Quand Dieu souffle.
}

Vent, respiration et notion de personne chez les Diola-Adiamat (GuinéeBissau)

André Julliard

\section{OpenEdition}

1 Journals

Édition électronique

URL : http://journals.openedition.org/assr/20217

DOI : $10.4000 /$ assr. 20217

ISSN : $1777-5825$

Éditeur

Éditions de l'EHESS

Édition imprimée

Date de publication : 1 septembre 2000

Pagination : 7-24

ISBN : 2-222-96695-7

ISSN : 0335-5985

Référence électronique

André Julliard, «Quand Dieu souffle. », Archives de sciences sociales des religions [En ligne], 111 | juilletseptembre 2000, mis en ligne le 19 août 2009, consulté le 03 mai 2019. URL : http://

journals.openedition.org/assr/20217; DOI : 10.4000/assr.20217

Ce document a été généré automatiquement le 3 mai 2019.

(C) Archives de sciences sociales des religions 


\section{Quand Dieu souffle.}

Vent, respiration et notion de personne chez les Diola-Adiamat (GuinéeBissau)

\section{André Julliard}

1 De manière générale, une religion de terroir ${ }^{1}$ fait coexister l'idée d'un Dieu unique avec une activité religieuse à caractère polythéiste qui s'appuie sur un ensemble (ou la combinaison d'ensembles) de puissances surhumaines ou de dieux établis ou non en panthéon. Ce Dieu unique est le créateur du monde dans la totalité de ses parties visibles et invisibles. Il n'est jamais personnel car Il se situe au-dessus du monde sans en être complètement extérieur. Il continue d'agir sur sa création même s'il se tient à distance des malheurs humains. Il n'a « besoin » d'aucun culte puisque son éloignement le rend inaccessible aux humains ${ }^{2}$.

\section{Le chef qu'on ne connaît pas}

2 Au nord-ouest de la Guinée-Bissau, le Dieu unique des riziculteurs-cueilleurs de vin de palme $^{3}$, Émitay $^{4}$ possède au moins trois caractères sociologiques connus des hommes et des femmes, des jeunes et des vieux :

3 1) Il est le Dieu des ancêtres, à savoir, ceux qui ont reçu palmeraies et rizières grâce auxquelles ils ont pu fonder les lignages actuels. Sur l'histoire de cette première occupation, les opinions divergent suivant la classe d'âge de l'interlocuteur. Schématiquement, les plus âgés qui ont peu de relations avec les sociétés environnantes défendent la tradition du Dieu qui les a fait naître sur les bords de l'Atlantique, tandis que par l'école, les émissions culturelles radiophoniques et le travail saisonnier en ville, les plus jeunes colportent les thèses d'une migration Diola par l'Ouest ou par l'Est depuis le nord de l'Afrique. Cependant, ils s'accordent pour conclure que premiers hommes ou premiers occupants, Dieu leur a octroyé ce territoire à charge pour eux de le faire fructifier. Aujourd'hui encore, les Ajamat retirent trois enseignements de cet héritage ${ }^{5}$ :

4 - n'étant pas propriétaire de la terre, ils se savent les hôtes permanents de Dieu ; 
5 - par cette relation contractuelle, les pères de la société étaient proches de Dieu. Cette proximité les contraignait à observer rigoureusement ses lois morales (absence de sorcellerie, d'adultère et de conflits armés, respect de l'autorité des anciens) en contre partie desquelles, Il favorisait l'abondance des récoltes. Restaurer les conditions de cette proximité, constitue toujours l'idéal social ;

6 - locataire de la terre, idéal de parachever le monde fini, devoirs et obligations de fertilité, constituent autant d'éléments sociaux, donc concrets, communs aux ancêtres et à leurs descendants. À l'Ajamat d'aujourd'hui, ils permettent de penser que le Dieu unique entretient avec lui les mêmes relations que par le passé ;

7 2) Émitay yoyu atia, «Dieu est en haut », et on conforte l'exclamation en pointant de son index le ciel. La place haute n'est pas une abstraction car le savoir ordinaire le positionne " collé à ulen au ", derrière la face cachée de la lune. Et malgré quelques témoignages contraires, elle ne constitue pas sa demeure : Il se tient là sans autre nécessité apparente que la volonté d'être au-dessus de la terre. Tout se passe comme si seul le caractère nocturne dont la face obscure de l'astre représente l'état achevé, permettait de concrétiser son immatérialité et sa puissance ontologiques. Quoi qu'il en soit, la majorité des Ajamat savent qu'il ne descend pas parmi les hommes : autrefois, me confiait en 1997 un gardien de bëkin, on ne disait jamais que Dieu est en bas ${ }^{6} . \grave{A}$ l'image de la hauteur, le langage courant préfère celle du lointain : Émitay éréjé, entend-on le plus fréquemment, "Dieu est loin ». Le très loin indique une inaccessibilité qui résulte moins d'une distance que d'une condition : Émitay est d'abord et surtout, « le tout autre », celui qui ne peut être connu par la pensée ;

8 3) On ne peut pas prononcer son nom. Or, il faut rappeler l'importance du nom qui, dans les sociétés lignagères en particulier, n'est pas seulement la traduction orale de l'objet ou du sujet. Il désigne leur nature même : il est l'objet ou le sujet.

9 Cette difficulté est plutôt d'ordre intellectuel que de l'interdiction car collectivement ou individuellement, l'Ajamat fait volontiers de nombreuses tentatives dans ce domaine. Les véritables interdits religieux (ñiñi, le sacré) s'appliquent au biologique tels les processus de la naissance ou encore les mécanismes physiologiques. Il est dangereux et souvent mortel de voir ou toucher l'énergie créatrice en cours de diversification dans l'homme.

10 Les deux propositions étymologiques que j'ai pu jusqu'à présent réunir, le soulignent parfaitement. Le pays Ajamat appréhende Émitay par une périphrase brève et précise : à la manière d'un chef [de groupe] qu'on ne peut pas connaitre ${ }^{7}$. Ésana $a^{8}$, en revanche, recourt à un détour plus lourd: attends [toi, que] j'appelle à nouveau sans te connaitre [et dont] personne ne peut [me] citer le nom. Cette étymologie en forme d'invocation, est particulièrement riche de sens. L'adverbe « à nouveau » introduit une redondance qui sursignifie le nom de Dieu : le prononcer dans quelque situation que ce soit, c'est chaque fois l'appeler. De son côté, l'impératif du verbe attendre par lequel j'ai traduit le préfixe "é» de Émitay, exprime un fort désir suscité par une première entrevue :

11 - ici, il pourrait s'agir de celle qu'ont connue les premiers hommes;

12 - de rechercher une deuxième rencontre 9 .

13 Conclusion importante : la puissance divine ne saurait être assimilée à un élément naturel qui ferait de Émitay le maître de la pluie ainsi que l'ont proposé plusieurs auteurs ${ }^{10}$. En effet, les Ajamat distinguent les gouttes d'eau (kusotaku) de la pluie (usotau : « il pleut une fois ») qui les suit : distinction tout à fait descriptive puisque le premier nom n'est que la forme plurielle du second. 

chaque fois le modèle de la puissance physique achevée: force dépouillée de toute violence passionnelle, de tout intérêt personnel, et canalisée au service unique de la communauté villageoise. En effet, dans les deux biographies, ses prouesses guerrières ripostent invariablement aux agressions extérieures qui menacent l'intégrité territoriale (la terre prêtée par Émitay) ou les capacités de reproduction du groupe (la mission de fertilisation). Bref ! Il intervient en quelque sorte contre les périls ultimes qui peuvent provoquer l'anéantissement du village. Dans le premier cas, il combat les villages voisins qui raptaient les adolescents d'Ésana; et dans le second, il affronte les maquisards du 
PAIGC qui, n'ayant pas sollicité l'accord du roi (sacrifice sur son autel) pour opérer dans la forêt, ont été assimilés à des meurtriers. Le guerrier-héros laisse à ses ennemis tout le temps nécessaire pour renoncer à l'attaquer. Contrairement aux coutumes de guerre, il n'a pas besoin de les intimider longuement et bruyamment pour les faire fuir, car sa victoire est indubitable.

Parce que ses sentiments pour l'ennemi demeurent impénétrables; parce qu'il reste impavide aussi bien dans la possession de la force que dans le moment de l'acte guerrier et le triomphe, Anbòna imite l'attitude de Émitay qui n'agit jamais sous l'emprise du courroux. "Dieu est lent à la colère», disent les Ajamat non sans admiration pour ce comportement que les hommes ont difficulté à tenir. Mais une fois force et colère "lâchées ", chacun frappe implacablement dans une totale liberté de gestes. Ils ne sont tenus par aucune règle morale ni contrainte coutumière. Ils sont guidés autant par le désir de victoire, que par le type de message qu'ils entendent donner à leur intervention. Ainsi, le premier rase tout ou partie du village vaincu ou s'empare d'autorité de leurs autels. Et le second emprisonne dans la lune une femme (relief lunaire) qui n'a pas respecté l'interdiction de sortir du village le lendemain du sacrifice demandant le retour des pluies.

La force achevée du guerrier-héros se compare à la colère de Dieu dans la mesure où l'une et l'autre manifestent une totale liberté d'être. Elles sont leurs caractéristiques inaliénables sans lesquelles le premier ne pourrait être un chef reconnu et le second le Dieu unique.

\section{La parole, expression de la force}

Si Émitay transcende incontestablement l'ordre du monde, il est non moins incontestable qu'il est immanent à la vie sociale. Ce constat ne relève pas du paradoxe pour l'Ajamat car il ne fait que rapporter le sentiment général de la très grande indisponibilité de Dieu pour tout ce qui concerne le quotidien de l'homme. C'est pourquoi sa position demeure une énigme. Il se place au-dessus mais pas à l'extérieur du monde. De même, Il se pose à distance des affaires humaines sans pour autant être étranger à leur devenir. La lune ne saurait cacher sa sensibilité à l'égard de l'histoire des hommes !

D'un autre côté, l'immanence de Dieu n'est pas un regard pesant à l'affût des errements humains. Elle tient de l'écoute disponible plutôt qu'inquisitoriale, vigilante plutôt qu'insistante. Elle repose sur le système des ukin ${ }^{14}$. Au moment du sacrifice, « Dieu envoie le bëkin » - Émitay éboño lòpi bëkinabu - qui convoiera le message des hommes (demande de protection, de bénédiction, d'intervention ou de jugement). En même temps, le réseau dense des autels offre autant de " cibles » [par lesquelles] «Émitay sait où se trouvent les hommes qu'il entend parler de lui», selon le desservant du principal autel de la circoncision qui expose l'opinion silencieuse d'une majorité d'hommes et de femmes d' Ésana. Un Ajamat occupant un poste dans le gouvernement bissauguinéen, ajoute ce commentaire en français (1994) : « Dieu laisse traîner ses oreilles dans le bëkin ».

Dans les deux cas, Dieu diffère toujours sa réponse - quelquefois de plusieurs années - et surtout peut l'adresser à n'importe quel villageois adulte. «Il descend pour parler avec toi ", Émitay éualu mané élorau, par le truchement des rêves dans lesquels Il apparaît sous la forme d'une lumière blanche, d'un vieillard aux cheveux blancs ou de l'un ou l'autre des desservants d'ukin. Dieu profère alors de courtes phrases. 

sa rareté. Prenons cette histoire où, au début de l'hivernage (fin juin) 1984, une femme revenant des rizières croise un rat palmiste qui au lieu de s'enfuir dans la forêt, trottine devant elle jusqu'à l'entrée du village. Informé, le roi la convoque pour qu'elle relate complètement l'affaire en versant une libation sur son autel dont la puissance (surhumaine) confirme la volonté de Dieu de parler aux villageois. Arrêtant le travail dans les champs, ils se réunissent pendant une journée dans le kayaku, bëkin royal : les hommes et les femmes (assises devant l'entrée de l'aire sacrée) exposent librement leurs propres interprétations de l'événement. Au moment du sacrifice d'une chèvre et de deux poulets, le message est alors très clair pour les gardiens de ce bëkin et notamment pour cet adjoint du roi : «Émitay est fâché d'entendre de la musique tous les soirs [bals organisés par les jeunes] alors qu'autrefois on n'entendait que les lames de kayendo ${ }^{15}$ dans la nuit ». Dieu et les adultes sont courroucés de voir les forces jeunes se disperser dans les danses au lieu de se concentrer dans l'effort collectif de labour des rizières. Travail et loisir sont des temps séparés pour l'adulte responsable de famille. Compte tenu de la désertification progressive de la région, son temps est compté pour rendre la terre fertile et pour ce faire, il doit mobiliser toute son énergie et son endurance physique. Une activité que les générations d'après-guerre de la libération nationale, ne considèrent plus comme un devoir mais comme un travail parmi d'autres, notamment ceux qu'offrent les centres urbains.

Rêve et événement significatifs constituent chacun une parole divine qu'il faut chaque fois rendre audible à l'entendement humain. Une tache ardue qui requiert souvent l'effort conjugué de plusieurs modes d'interprétation, donc d'intervenants différents (divination, mémoire des anciens, expérience individuelle, savoirs naturalistes) pour accéder au sens global. Dès lors tout le village a conscience que l'explication retenue n'est exacte que parce qu'elle est la plus probable et jamais parce qu'elle est certaine.

Aussi, le courroux de Émitay n'a pas été compris de la même manière par tout le monde. Seules les classes d'âge les plus anciennes ont dénoncé les pratiques inhabituelles des «bals d'hivernages». Les adolescents qui ont contesté, parfois violemment leur interdiction, avaient une autre version de l'affaire: Dieu réclamait le sacrifice pour l'abondance des pluies qui effectivement n'avait pas été accompli au début de la saison des pluies. Ils ont mis en cause les « vieux » qui ont préféré retarder la cérémonie plutôt que de perdre une journée de travail. De leur côté, les adultes ont plutôt retenu un avertissement adressé à l'ensemble des villageois qui ont tendance à laisser en friche les rizières pour un emploi salarié dans les capitales régionales de la Guinée-Bissau ou du Sénégal voisin.

29 Au-delà de l'événement social rapporté, cet exemple illustre combien l'écoute de la parole de Dieu est un exercice ardu et complexe. La plupart du temps, elle n'est entendue ni sur les lieux (ukin), ni dans les moments, ni par les personnes dont les fonctions assurent officiellement la communication avec Émitay. Par conséquent, les autorités religieuses doivent chaque fois l'authentifier (sacrifice et divination) pour qu'elle soit reconnue comme message divin. Mais leur interprétation ne fait pas automatiquement l'unanimité. D'autres instances du social (classes d'âge, groupe des femmes mariées, le roi, etc.) peuvent proposer leurs propres traductions qui, notons-le, se complètent rarement sans pour autant se nier les unes les autres.

30 En quelque sorte, la parole divine surgit au moment où persiste un malheur collectif (raréfaction des pluies; brusque progression de la mortalité; épidémie; etc.). 
Manifestation de Dieu, la persistance du malheur met le village en attente de sa parole. Lorsque cette parole survient enfin, elle révèle a posteriori une affaire (ici, le refus du sacrifice pour la pluie) dont l'importance n'a pas, sur le moment, été saisie partout le monde. Les interprétations de la parole de Émitay opposent donc des groupes sur la reconnaissance précise des causes et la délimitation des responsabilités respectives. Chacun d'eux tient son interprétation pour le contenu global du message alors qu'elle ne commente qu'une information: sur la contingence des rites, l'arbitraire des lois ou encore l'importance de la morale sociale, pour rester dans notre histoire. Au total, le degré d'opposition et de contradiction entre les informations retenues, n'est que l'indice de la gravité du message: l'essentiel du contenu reste hors de portée des efforts de compréhension.

Dans l'événement cité, lors du sacrifice final dans le bëkin royal, l'affrontement des trois informations a obligé le village à prendre des mesures sévères: interdiction de toute musique jusqu'à la récolte du riz, sacrifice d'une poule noire par maison et deux jours d'arrêt des travaux agraires pendant lesquels personne ne pourra sortir du village. Cette fois, le partage des responsabilités renvoie dos à dos les vieux qui n'ont pas respecté l'ordre des sacrifices et les jeunes qui ont contesté le devoir collectif de fertilité.

Au-delà du rappel à l'ordre, parfaitement compris même s'il fait débat, qu'a voulu dire Émitay? Personne ne peut répondre bien que tout le monde sache que le contenu était, cette fois-ci encore, plus déterminant que l'avertissement. Il y a "un savoir profond" logé au cœur de la parole qui déclenche - en raison même de son impénétrabilité - la forte charge émotionnelle unanimement ressentie. Charge que l'on peut concrètement appréhender ne serait-ce que par les sentiments immédiats qu'elle suscite: angoisse, anxiété, espoir et contentement. Ensuite, son intensité se mesure aisément à «la participation aux mots» qui implique jeunes, vieux, hommes, femmes, convaincus et sceptiques. Enfin et à défaut de pouvoir analyser son contenu, nous pouvons imaginer sa teneur à partir du style de l'élocution. Quel que soit son mode d'expression (rêve, événement, etc.), la parole de Dieu est toujours ramassée, épaisse et lourde de silence (le vrai langage de Émitay). Elle ne saurait alors manifester autre chose que la puissance souveraine de la Loi qui, aujourd'hui, intime aux villageois charges (faute, erreur ou oubli) et commandements (sacrifice, libation, etc.). Mais dans le moment de l'écoute, le moment des interprétations officielles et individuelles, l'Ajamat n'a jamais été placé aussi près du « texte de loi » puisqu'entre les deux il n'y a plus les médiations ordinaires: les interdits, les obligations et les rituels. En même temps, il n'a jamais été aussi éloigné d'en prendre connaissance car, nous l'avons vu plus haut, la condition à remplir est le parachèvement collectif $\mathrm{du}$ monde (éradication de la sorcellerie, respect des règles sociales, etc.). C'est pourquoi, l'un des guerriers du roi peut à la sortie du sacrifice, me dire sans contradiction que Émitay ne parle pas à l'Ajamat, Il ne lui dit pas l'essentiel !

La parole de Dieu répète que la Loi existe mais ne la divulgue jamais. En face, l'Ajamat la pense par une parole longue, continue et pleine de détours métaphoriques qui ne fait que constater la faiblesse de la condition humaine. Pour le premier, elle n'est qu'efficacité tandis que pour le second, elle est surtout problème. Élobay, la parole, s'emploie dans ce deuxième sens pour signifier toute controverse d'ordre moral : du différend entre époux au questionnement religieux. 


\section{Éntélay, le souffle de Dieu} de ces comportements, l'arrivée du vent, éntélay, provoque encore l'émoi de toute la population: de la fuite des enfants aux cris des parents! En février 1993, une forte bourrasque de poussière rouge s'engouffre dans une rue de Ésana où trois femmes s'apprêtaient à entrer dans une boutique. En entendant le grondement, elles s'allongent précipitamment face contre terre en chantant : " pardon, pardon, pardon / continue ton chemin, je n'ai pas gâté quelque chose à toi/continue ton chemin et moi je continue le mien ». Elles garderont cette position défensive et conjuratoire jusqu'à la complète disparition de la menace. Peu après, l'une d'entre elle commente son comportement: «lorsqu'il y a un vent de poussière (...) le vent qui vient avec un souffle qui dit tou, tou, tou (...) il faut se coucher par terre pour que la maladie passe au-dessus de toi. Si tu as un panier, tu le poses devant toi et tu t'aplatis et dis : uasi, uasi, uasi, piru, piru, piru, pardon, pardon, pardon, passe, passe, passe ».

- il y a ceux qui, nous l'avons vu plus haut, venant de la mer et plus exactement de l'estsud-est où celle-ci remonte dans les terres par un lacis de marigots (estuaire du Rio $\mathrm{Cacheu}^{16}$ ), apportent les meilleures pluies, Émitay ésòtolò, pour les rizières. Ils n'ont pas de nom générique propre. Celui du moment se nomme en accolant le nom de Dieu à celui du village côtier qui localise sa direction: par exemple Émitay Élalam, île qui, une fois par semaine, ravitaille le bourg en poissons frais. On remarque que la similitude du procédé de nomination ne s'applique qu'aux pluies et vents dont l'action dépourvue d'ambiguïté est uniquement et obligatoirement bénéfique. Plusieurs témoignages, hommes et femmes, considèrent que l'on remercie Dieu « d'envoyer à manger à Ésana »;

- pendant l'hivernage, bututénorabu, la tornade est ainsi appelée par homophonie car en se déplaçant, elle fait un bruit saccadé: butu, butu, butu. Elle est redoutée pour ses dévastations forestières qui font notamment craindre l'effondrement des grands kapokiers ${ }^{17}$ sur les cases ;

38 - le vent chaud et sec qui, lui non plus, n'a pas de nom, souffle au cœur de la saison sèche (avril-mai). Lorsqu'il sévit trois jours de suite, il annonce le décès d'un personnage important (homme ou femme) : un roi, un vice-roi ou l'un des gardiens du bëkin royal ( kayaku), de la forge (karañaku) ou des femmes (karayaku);

39 - en début de saison sèche (janvier-février), surviennent souvent des vents de poussière qui apportent des maladies bénignes (traumatismes nasal et oculaire) ou mortelles (épidémies). Toujours sans nom, ils surgissent de l'intérieur des terres guinéennes (nordnord-est) ou sénégalaises (nord-ouest);

- enfin, la plupart du temps, l'activité éolienne ne fait l'objet d'aucune attention particulière. Dans ce sens, il faut noter qu'à Ésana, on préfère employer le générique buntélabu qui est la forme plurielle de éntélay. «Les vents » désignent ces circulations de faible amplitude qui, du fait de la proximité de la mer, balaient fréquemment la forêt. Ils ne sont pas inquiétants car ils régulent « en surface » la température de la terre. De son côté, éntélay signifie également l'air conçu comme la matière du vent: son corps matérialisé qu'il laisse après son passage (pour les nécessités respiratoires de l'homme) et qui lui permet de transporter « une chose » telle la maladie.

Archives de sciences sociales des religions, 111 | juillet-septembre 2000 
41 L'absence de nomination des vents est d'autant plus remarquable que les Ajamats ont le souci d'appeler chaque objet ou chaque être vivant par autant de noms qu'il compte non seulement de caractéristiques mais aussi de modifications possibles ${ }^{18}$. De plus, aucun d'eux n'est considéré comme une puissance surhumaine : il n'y a pas à ma connaissance de bëkin pour éntélay. Qualifié uniquement par l'une des trois fonctions: régulation calorifique, apport de pluies et porteur de maladies, chacun d'eux est identifié exclusivement à une force participant au jeu de celles qui animent l'univers. C'est pourquoi, leur survenance inquiète et effraie toujours même si pour certains, ils sont désirés et attendus. Quelles sont alors les caractéristiques de ces forces?

En avril 1992 alors qu'un tradipraticien me décrivait les vents, une veuve âgée en traitement thérapeutique à demeure, interrompit notre conversation : "c'est Dieu qui envoie les vents et personne ne sait comment il fait». Son intention était claire : elle voulait le faire taire en lui rappelant le danger qu'il encourt à vouloir expliquer les actes de Dieu et de surcroît à un étranger. Cette attitude commune aux gens âgés, certifie en même temps l'opinion commune : la force éolienne dépend directement de Dieu.

43 Cependant, pour les classes d'âge adolescentes et adultes d'aujourd'hui, il n'y a pas vraiment d'interdit sur ce sujet car en réalité, il n'entre pas dans les conversations quotidiennes des Ajamats. Donc, en dehors des "vieux " qu'on ne veut pas offenser, on peut préciser d'autant plus librement sa pensée que tout le monde rejoint leur conviction : l'homme (noir ou blanc) ne saura jamais comment Dieu fait le vent ! Cette certitude est la meilleure assurance de rester toujours à distance de son œuvre.

Au total, trois types d'explications résument la diversité des commentaires sans que l'une ou l'autre puisse être attribuée à un groupe social particulier :

- «le vent est la respiration de Dieu », affirme clairement Jaméjo, une jeune femme divorcée à Ziguinchor et récemment remariée à Ésana. Elle fait figure d'exception car cette analyse s'entend très rarement en dehors d'un petit cercle d'hommes entrant dans la vieillesse et qui n'appartiennent ni au même segment de lignage, ni au même quartier et qui, à l'exception d'un seul, ne sont pas initiés à des grands ukin. Ils partagent le seul point commun d'être certainement dans cette classe d'âge, les plus ouverts sur les sociétés extérieures : fréquents déplacements à Bissau et à Ziguinchor (Sénégal) ; enfants étudiant ou travaillant dans les capitales régionales et européennes. Parmi eux, le tradipraticien défenseur de la participation des ukin à certains vents, généralise avec assurance : " ils sont créés par Dieu qui souffle comme nous, nous soufflons » (dans le sens de respirer) ;

46 - il est un flux d'air produit par un instrument assimilé soit à « une pompe à vélo » soit le plus souvent, à un soufflet de forge ${ }^{19}$. Pour le plus grand nombre, Dieu actionne lui même cet instrument : " le vent n'est pas le souffle de Dieu, Il fait comme avec un éventail ", nous disait un adolescent en 1992. Alors que pour une petite minorité, son fonctionnement autonome obéit à son ordre. Tout développement complémentaire s'avère difficile voire impossible à obtenir : on entre alors dans l'inconcevable ;

47 - enfin, "c'est Dieu qui souffle parce qu'il est fâché ", selon deux témoignages féminins concernant la tornade ${ }^{20}$. Toujours pour exprimer son courroux, Jaméjo nous dit que « des fois quand il y a des nuages et que Dieu ne veut pas faire pleuvoir, Il souffle du vent et on voit de fines gouttes et les nuages se dispersent ». De même, pour le desservant du bëkin qui préside à l'installation de tous les autres ukin : « Dieu souffle le vent chaud et sec » qui, en réchauffant la terre, menace les cultures et la vie des hommes. 
Finalement les deux points de vue les plus répétés à Ésana, assimilent le vent à un acte (technique du soufflet) ou à une action (expulsion d'air) de Emitay. L'Ajamat navigue de l'un à l'autre sans contradiction ni débat théologique: la priorité est donnée à l'interprétation immédiate et urgente des intentions divines. Mais quel que soit le raffinement des connaissances individuelles, il est clair pour le plus grand nombre que le vent envoyé aux hommes est « du fait » du souffle de Dieu.

Ma conclusion est prudente car il semble que la représentation d'ordre instrumental s'impose surtout par défaut de pouvoir mener à terme celles qui sont d'ordre physiologique. Encore une fois, discuter de la parole ou de la respiration du divin, relève d'un exercice non seulement vain mais aussi socialement dangereux. «Crois-tu que je suis un bëkin pour aller voir si Dieu a une bouche? Même les Blancs qui construisent des avions n'ont jamais vu Dieu ", me répond ce tradipraticien qui avait déjà refusé de me détailler l'organisme humain sous prétexte que n'étant pas un assassin, il n'a jamais dépecé de cadavre. Montrer des connaissances sur les poumons, le sang, le cœur suscite la méfiance d'autrui car c'est quasiment avouer être " un homme méchant » c'est-à-dire une personne dont le double se déplace dans l'invisible pour dévorer ceux des villageois. Il transgresse quotidiennement les interdits et notamment celui de voir l'intérieur des corps aussi bien masculins que féminins. Systèmes et processus biologiques constituent le sacré chez les Ajamat: le biologique divin qui se confond avec l'énergie fondamentale de la création, s'érige alors en un interdit que ne peut percer aucune pensée humaine.

contre, s'il participe sans conteste de l'énergie divine, il n'est que le produit du travail respiratoire. Le vent représente cette force qui est la forme de la création continuée sur terre. Mais détachée, éloignée de Dieu, elle devient un objet pensable sans périls excessifs. C'est pourquoi, le jugement commun le perçoit comme un instrument du divin et résume sa fonction principale : «le vent porte la parole de Dieu ». Il transporte avertissements, condamnations et envois de fertilité sous forme de charges de chaleur, de pluie ou de maladie. Seules les deux dernières font l'objet de commentaires, peut-être parce qu'elles concernent plus systématiquement les intérêts vitaux du village.

Beaucoup d'hommes, âgés ou non, lient par observations empiriques la pluie aux marées d'un océan qui imprègne la terre à plus de $60 \mathrm{~km}$ de ses côtes. Le vent se charge d'eau à marée haute, mais le chargement n'obéit pas aux seules règles écologiques. C'est également le choix de Dieu parce que, ajoute Jaméjo, « il y a aussi les gouttes qui viennent d'Eramé et qui amènent des maladies ». Autrement dit Émitay Éramé, la pluie venant de ce petit village de la côte est, contredit sa classification dans la catégorie des précipitations uniquement fertilisantes puisqu'elle peut être néfaste à la santé humaine. L'eau de mer n'est pas bonne ou mauvaise en elle même : elle le devient selon ce que Dieu veut dire aux hommes.

Aussi l'argument du châtiment fournit la première réponse. "Dieu envoie le vent des maladies », m'expliquait en 1992 un tradipraticien "parce qu'une fois que les hommes ont fait les sacrifices à tous les ukin [calendrier rituel] ils en ont sûrement oublié un. Alors ce bëkin demande à Dieu d'envoyer le vent des maladies et s'il le fait, c'est que c'est un grand bëkin. Mais l'explication n'est pas entièrement satisfaisante car elle ne s'applique pas à toutes les situations. Et mon interlocuteur devance les objections en poursuivant: « moi avec ankurénau je peux le voir [dans l'invisible] et lui dire qu'Ésana reconnaît son erreur ». Apaisé par un sacrifice réunissant tout le village et plus conséquent que ne le requiert le rite, il demandera à Dieu de suspendre la sanction. Si le vent apporte des maladies, conclut-il, c'est lui qui les chasse en les emportant dans la mer. Peu de jours après, au 
cours d'une nouvelle discussion avec deux de ses frères également détenteurs d' ankurénau, ils convinrent ensemble que d'autres motivations peuvent conduire l'action divine. « Dieu envoie des maladies une année et l'autre année II les diminue », m'apprendil tandis que son frère le plus jeune conclut : « c'est vrai, le vent apporte des maladies, il apporte aussi la mort. C'est Dieu qui envoie le vent pour faire diminuer le nombre des hommes pour qu'il puisse augmenter les naissances l'année suivante. C'est comme avec les pluies qu'il donne puis II les enlève pour mieux les redonner ». Par la suite, je n'ai entendu cette explication que chez les hommes âgés de ce lignage dont la plupart sont responsables des plus importants ukin du village.

La survenance des vents même désirés et attendus, inquiète et effraie car ils convoient toujours des interventions de Dieu qui ne sont pas en nombre illimité. Autrement dit, ils diversifient l'énergie divine de cinq manières différentes : 1 ) en soutenant la respiration humaine (renouvellement de l'air); 2) en distribuant l'énergie fertilisante (rafraîchissement de la terre, apport de pluies) ; 3) en signifiant son mécontentement (assèchement ou réchauffement de la terre) ; 4) en appliquant des sanctions (maladies); 5) en régulant la démographie (épidémies). Quelle que soit sa fonction, éntélay étreint l'intérieur de l'être, obligeant pareillement tous les individus présents, à participer aux mouvements de sa force. Saisissement et participation dégagent l'émotion par laquelle "l'homme concret, vivant, accède au sens profond du monde, non pas par sa seule raison, par abstraction, mais par son être total, corps et esprit liés ${ }^{21} »$. Sa participation immédiate mêle savoir écologique (géographie du territoire, circulation cardinale des vents, horaire des marées, contexte saisonnier, etc.), sensations corporelles (évaluation de la chaleur, la sécheresse, l'humidité, etc.) et sensibilité religieuse.

\section{La respiration du corps}

Le vent saisit l'être dans sa globalité où la distinction avec le corps n'a aucune pertinence. Une pensée biologique soutient la notion de personne (anau) qui met en relation constante les déterminismes de l'hérédité (caractères moraux et physiques donnés par Dieu, reçus des parents et des ancêtres), le fonctionnement bio-physiologique du corps et les bouleversements écologiques et sociologiques - souvent très rapides - de l'environnement quotidien. Donc, elle est aussi une pensée de l'identité individuelle en devenir.

L'Ajamat repère six composants de anau :

- le corps physique (éniley) comprend une enveloppe, kakoñaku qui reste après la mort et un organisme intérieur que nous détaillerons plus bas ;

57 - le sang (la sève : asimau) est également un composant à part entière de la personne. Ce n'est pas son importance vitale à la survie biologique qui lui accorde cette place : d'autres organes (les poumons) ou liquides (eau) pourraient alors prétendre l'occuper. Son statut vient de son autonomie et de sa mobilité à l'intérieur de l'organisme. Donnée par Émitay à part égale à tout être conçu, quel que soit son sexe, cette quantité de sang doit sans cesse être augmentée au cours de la croissance et maintenue " à niveaux » à l'état adulte et lors de la vieillesse. Chaque individu doit veiller à son développement en respectant des règles d'hygiène tant alimentaires (consommer le plus souvent possible de l'huile de palme et boire uniquement de l'eau du puits) que morales (éviter de répandre le sang d'autrui ; réserver sa force à la riziculture ; etc. $\left.{ }^{22}\right)$; 
58 - la renaissance (jamais une réincarnation) de quelques caractères physiques ou moraux d'un défunt, parent ou familier de la maison. Ils agissent plutôt sur le comportement social du renaissant (auau);

59 - le principe vital, yarorey, est matérialisé par la silhouette, ombre portée au sol de l'individu. Chaque homme ou femme, chaque enfant, adulte ou vieillard en compte entre cinq et sept, voire plus pour des êtres exceptionnels tel Anbòna. Quelques-uns d'entre eux sont spécifiques d'une fonction: la clairvoyance c'est-à-dire la capacité de voir et de se déplacer dans l'invisible; la malfaisance dans le sens d'une aptitude qui pousse son détenteur à dévorer le principe vital des villageois; ou encore, la renaissance. Ainsi, un être malfaisant, un être qui «bouffe » les gens (les deux ont été assimilés à des sorciers dans la littérature ethnologique) et un revenant s'appellent respectivement au yarorey, asayao yarorey et yarorey. Pour certains tradipraticiens, les deux ou quatre yarorey supplémentaires, constituent des sortes de bouclier contre les malheurs biologiques. Chaque fois que «toi tu passes près de la mort et que tu restes en vie, c'est un de tes yarorey qui s'en va (...) quand il t'en reste plus, tu vas vraiment mourir ", me confiait l'un d'entre eux en 1986 ;

60 - le double, éumey, est un animal aquatique (hippopotame, lamantin, marsouin) ou terrestre (biche, panthère). Tout ce qui touche éumey se répercute sur la personne et réciproquement. Comme les yarorey, ils sont au nombre de cinq ou sept, donnés par Émitay à l'enfant dès sa conception et leur croissance est en prise directe sur celle de l'individu : elle sera fonction de son comportement social. À la différence des premiers, les doubles de tous les villageois, s'organisent en société dans un lieu inconnu: par exemple, sous le fond des marigots. Yarorey et éumey permettent à l'individu d'agir dans l'invisible soit de manière active et consciente, soit de manière passive et en quelque sorte « malgré soi ». Le premier accentue les déterminations singulières et contraignantes du sujet ("être malfaisant», clairvoyant, etc.) tandis que le second participe des caractérisations sociales indépendantes de la personne ;

61 - l'esprit (buyagitabu), la raison (buinum) confèrent l'aptitude intellectuelle à mettre en correspondance l'ensemble des données concernant l'hérédité, la biologie et la sociologie. L'homme se distingue ainsi de l'animal parce qu'il peut penser individuellement son destin.

62 Comment le souffle de Dieu anime-t-il la personne Ajamat? Très concrètement, en la faisant respirer: "le vent c'est ce qui permet à l'homme de continuer à respirer ", confirme le responsable d'un bëkin ankurénau. L'un de ses cousins paternels, décrit par ailleurs le processus avec réalisme : «Émitay nous pompe du vent dans le corps comme on fait pour gonfler les pneus d'une voiture, c'est ce qui nous fait vivre ». Et ce n'est pas la première fois qu'on utilise la métaphore de l'automobile pour appuyer la description du processus. « L'homme est fait par Émitay comme une voiture. Quand tu cours jusqu'à Kagit [environ 40 kilomètres] tu ne peux plus respirer. Il faut de l'air pour parler. Émitay a mis dans la gorge deux tuyaux [il montre une paille creuse et la trachée artère] qui descendent dans le poumon. Quand les tuyaux se bouchent, l'homme meurt " (1988, détenteur du bëkin qui sert à la phase finale de l'initiation à cette fonction ${ }^{23}$ ).

63 La série de témoignages ne différencie pas expressément le vent de l'air. Éntélay constitue à la fois « le moteur et le carburant » du mécanisme respiratoire dont la parole fait partie intégrante comme le suggère ouvertement mon troisième interlocuteur ${ }^{24}$. Cependant, il semble que nous puissions déceler un schéma de fonctionnement où l'air, souffle continu 
de Émitay, alimente la respiration instinctive ( ... continue de respirer ») tandis que le vent, souffle périodique de force, recharge la machine humaine ( «...pompe le vent dans le corps »).

Mais personne ne pense que le corps se remplit d'air tel un ballon. L'image est faite pour la discussion publique où chacun dissimule soigneusement ses connaissances anatomiques de peur d'être au mieux désigné comme un guérisseur et, au pire, suspecté de «malfaisance ». Il connaît l'intérieur du corps parce qu'il dévore la nuit le principe vital des villageois. Seuls les tradipraticiens dûment institués acceptent en privé - mais pas en secret - de faire une description anthropologique.

L'organisme se concentre presque entièrement dans la poitrine (ésigirey) dont le cœur ( éjundé) occupe la place centrale, juste derrière le plexus solaire. Quatre autres organes s'agglomèrent autour de lui : l'œsophage (émérumey), l'intestin (mulaum), le poumon ( unuéu) et le foie (ubrikau).

Le sang est présent dans ce complexe de deux façons simultanées. «Il est dans tout le corps, selon un tradipraticien décédé en 1988, sous la peau comme une flaque d'eau mais il s'écoule aussi par les veines ». Et le vice-roi qui assiste à notre discussion, ajoute : «le sang circule de l'épaule au bout des doigts et il revient ». Quelques années plus tard, un autre tradipraticien nuancera cette vue : « il va des cheveux au gros doigt de pied mais il ne fait pas ce chemin tout le temps ».

Le sang n'entretient aucun lien physiologique avec le conglomérat organique logé dans la cage thoracique. Il a pour fonction première de circuler dans tout l'intérieur du corps sans qu'aucune de ses parties ne soit laissée à l'écart. Par ailleurs, il semblerait qu'il y ait communication entre l'enveloppe sanguine interne et les veines. La première sert-elle de réservoir d'où partent et arrivent les secondes? La question donne à réfléchir autant à l'ethnographe qu'à ses interlocuteurs! Cependant, tous sont à peu près d'accord pour admettre que cette «flaque » contient la quantité de sang donnée par Émitay lors de la naissance.

La tâche réservée à l'homme consiste "à faire augmenter » régulièrement son volume sanguin pour mettre en adéquation sa force physique avec les exigences de travail propres à chacune des étapes de la vie ${ }^{25}$. Cette augmentation se fait naturellement si l'on respecte la première des prescriptions alimentaires: «il faut boire de l'eau [des puits] parce que c'est l'eau qui fabrique le sang ou peut-être la fait circuler. On le voit parce que quand on se coupe, l'eau [la lymphe] sort avec le sang ». Le vice-roi ne fait que répéter un savoir maintes fois entendu des plus jeunes aux plus âgés. Pourquoi l'eau des puits estelle la seule capable de régénérer le sang? Il y a au moins trois explications qui ne s'excluent pas entre elles. D'abord, ce sont les seuls lieux où elle est potable dans ce pays de marigots aux eaux saumâtres. Ensuite, les puits dont la profondeur minimale est d'une dizaine de mètres, la mettent en contact avec la terre du sous-sol et non, comme les mares affleurantes, avec la terre de surface porteuse de « la poussière-maladie ». L'argile latéritique épure le liquide de tout goût terreux et salin : elle restitue une eau à la saveur minérale très désaltérante. Enfin, l'eau puisée à cette profondeur provient - et tous les vieux le savent - de la mer sur laquelle « est posée la terre où on est tous les jours assis pour discuter » avec l'ethnographe. Cette mer est en quelque sorte l'abîme de l'océan Atlantique où, rappelons-le, résident habituellement les ukin.

La poitrine contient l'essentiel de l'organisme humain qui ne se pense pas en terme de structure. Non seulement aucun ordre n'organise foie, poumon, cœur, œsophage, intestin 
et sang, mais ils n'ont aucune relation organique entre eux. Leur fonction individuelle est d'abord collective : participer au mécanisme de la respiration humaine (uiau).

En 1984, un tradipraticien présenté par le vice-roi, m’en révélait la subtilité : «éntélay [l'air] passe par le nez et descend frapper le cœur (...) c'est pour ça qu'il fait toujours du bruit [et que] tu sais que tu es en vie ». De son côté, la matrone de l'une des maternités traditionnelles du village, abonde dans son sens : « quand tu respires, c'est là que va l'air [elle montre le plexus solaire] quand ça se coupe tu meurs (...) ce n'est pas gros et ça fait tic, tic, tic ». Elle ne nomme aucun organe en particulier bien que sa dernière parole évoque sans aucun doute le cœur: ce qu'elle ne me confirme pas, répétant ésigirey, la poitrine. Mais, il est évident que les deux se rallient à la remarque du vice-roi : « le cœur fait respirer le corps ». Sous l'impulsion de l'air, le cœur déclenche l'élévation et l'affaissement mesurés du «bloc» des cinq organes et du sang. Ils entraînent la respiration signe de bonne santé.

71 Une respiration harmonieuse dépend de la constance de sa source d'alimentation (le souffle de Dieu) et en même temps de la stabilité de la chaleur interne dégagée par le travail mécanique du cœur. L'Ajamat redoute autant «le corps froid», celui qui ne bouge plus que « le corps chaud ${ }^{26}$ ", celui dont la chaleur interne entre en ébullition. Un corps à bonne température s'apprécie par plusieurs symptômes: la peau reste fraîche malgré l'ardeur du soleil et aucune douleur ne raidit la souplesse musculaire. « On est bien quand le sang bouillonne et va dans tous les bras et les jambes, là tu peux aller débroussailler longtemps sans te fatiguer ", selon les efforts d'un adolescent pour me décrire cet état.

La respiration contrôle la circulation sanguine qui passe par le cœur ${ }^{27}$, « là où le sang est frais " (le vice-roi). Le soulèvement et l'abaissement de la poitrine agitent le liquide sanguin qui lui est solidaire. L'amplitude monotone de l'agitation lui assure une température constante et une ventilation permanente, contribuant ainsi à sa bonne fluidité. L'essoufflement du mouvement entraîne le ralentissement puis la stagnation du sang qui progressivement s'échauffera et entrera en ébullition. Cette phase ultime constitue une peur majeure car le corps ne canalise plus la circulation. «Si tu te coupes, tu ne pourras plus arrêter le sang", nous dit l'adolescent tandis qu'une femme surenchérit : «le sang va monter dans le poumon [qui] va gonfler, devenir tout noir et monter dans l'œsophage ». En conséquence, le principe du chaud et du froid est, par rapport au corps, lié aux notions de travail organique et de force physiologique: un « corps froid » est d'abord et surtout un corps dont l'organisme interne étant à l'arrêt, se remplit de chaleur. Elle pourrit les organes et les transmute en liquides qui, s'échappant du corps, provoquent la mort biologique.

Le remède est connu de tous. Pour relancer les battements du cœur, «il faut boire de l'eau chaude » c'est-à-dire qui n'a pas subi de transformation mécanique telle son rafraîchissement dans un pot en terre.

Le rôle de l'eau dans le mouvement respiratoire, ne se limite pas à régler le niveau de sang en circulation et soutenir le rythme cardiaque. À l'inverse, elle peut endommager la mécanique de la respiration. En effet, elle véhicule de deux manières différentes, «la poussière-maladie » à l'intérieur de l'organisme. Soit par absorption de l'eau des puits qui a été contaminée par les vents de poussière rouge. C'est le savoir commun de Ésana. Soit parce que cette dernière traversant la poitrine, « frappe » une sorte de matière graisseuse et aqueuse (umpunau) qui enveloppe la cage thoracique. À ce niveau, le processus s'opacifie : la poussière se transforme en eau ou bien se mélange à celle contenue dans 
umpunau. C'est le savoir communiqué par trois tradipraticiens (deux hommes et une femme) mais qui, me semble-t-il, est possédé par l'ensemble de leurs confrères.

Quel que soit le trajet, le résultat est identique. L'eau-maladie « va tomber sur le cœur si Émitay veut donner cette maladie » (la guérisseuse) et par conséquent, sur l'un des quatre autres organes suivant le type de maladie envoyée. Elle l'individualise en lui faisant subir des changements de volume, de couleur et même d'odeur. Deux exemples: le foie et le poumon gonflent et noircissent tandis qu'une femme diagnostique son indisposition: «j'ai mal à l'intestin [parce que] ça sent mauvais dans le ventre ».

Mais surtout cette eau anime "l'organe-cible" d'un mouvement individuel, le plus souvent ascendant qui disloque l'agglomérat thoracique: foie, poumon ou cœur « remonte » dans la gorge et fait craindre l'étouffement ou l'expulsion hors du corps (la création divine devient visible) ; l'intestin s'enroule et se déroule sur lui-même de plus en plus vite. Bien entendu, chacun de ses états représente un degré dans la gravité de la maladie qui, notons le, est perçue comme une mise en ordre de l'organisme humain.

Le vent et l'eau ne sont pas les seules forces agissantes sur le développement respiratoire. Des sentiments sociaux violemment ressentis, produisent les mêmes effets de dysfonctionnement organique. À Ésana et même si les cas sont rares, on meurt aussi de colère, de honte et surtout de peur.

Dans chacune de ces situations étiologiques, le doublement ou le triplement de volume de l'organe constitue le symptôme récurrent. Pourtant, ce n'est pas lui qui menace le mécanisme respiratoire mais l'un de ses effets secondaires : le déplacement autonome et individuel qui risque d'obturer le conduit œsophagique. L'eau chargée de poussière ou le sentiment qui fait sortir l'organisme " hors de soi ", gonfle l'organe non plus avec de l'air mais avec de la maladie. En conséquence, il s'agit toujours du souffle de Émitay même si dans ce cas, l'intention annoncée, est une agression. Autrement dit, si l'air respirable est bénéfique, il porte en lui un risque potentiel ${ }^{28}$ : le gonflement arbitraire de tel ou tel organe. Recevoir le souffle de Dieu n'est donc pas sans danger comme le fait remarquer une femme : «si l'air vient jusqu'au foie, tu meurs ». Affirmation dont elle ne lèvera pas l'ambiguïté : le foie est-il la jauge maximale de remplissage de la cage thoracique ? Ou bien le simple contact avec l'air, va-t-il le faire augmenter de volume? Cette dernière interrogation renvoie alors à la conception d'un organisme aggloméré qui n'offre au souffle divin qu'un seul point d'impact (le cœur $\left.{ }^{29}\right)$ : une masse unique protégeant les cinq organes. L'un des premiers tradipraticiens rencontré en 1984 pense que umpunau sert à filtrer l'air. Sans cette protection, les vents de poussière rouge tueraient immédiatement toutes personnes traversant leur chemin.

79 Sensibilité, émotion, valeurs affectives et même, motivations sociales prennent vie par «la respiration du cœur». Sous le principe du chaud et du froid, elle combine circulation de l'air, du sang, de l'eau et des éléments de la terre. L'accord avec Dieu mais aussi avec l'animation énergétique globale du monde, se fait (entre autre) par éntélay sous la forme de l'air et du vent. Le souffle de Émitay est plus une force de vie qu'une force de création.

C'est cette force qui est requise par les tradipraticiens et desservants d'ukin lorsqu'au sortir du rituel thérapeutique ou d'une intervention dans l'invisible (sauvetage d'un double), ils pratiquent le kapulinaku sur le patient ou la victime. Ils soufflent sur le front, les yeux, le plexus solaire, le creux des omoplates ou sur les côtes, en chuintant l'air de leur bouche et en projetant (ou non) un peu de salive ou du jus de noix de cola 
préalablement mâchée. Le kapulinaku donne la vie en même temps qu'il replace la poitrine (le cœur?) sous la protection de Émitay.

\section{NOTES}

1. Parmi les ouvrages de synthèse les plus récents en français, citons à titre d'exemple : Albert de Surgy, Nature et fonction des fétiches en Afrique noire, Paris, L'Harmattan, 1994 ; ou encore, JeanneFrançoise VINCENT, Daniel DORY et Raymond VERDIER (dir.), La construction religieuse du territoire, Paris, L'Harmattan, 1995.

2. «La Nomination de Dieu », Afrique et Parole, 33-34, juillet $1977: 77$ et 82 (Bernard de RASILLY); 87 (Michel CARTERON).

3. Constituant l'un des 17 groupes linguistiques qui composent le peuple Diola (Sapir J. DAVID, A Grammar of Diola Fogny, Londres, Cambridge University Press, 1965, I-II), les Ajamat sont installés de part et d'autre de la frontière sénégalo-bissauguinéene. L'habitat se situe en forêt dans une zone de marigots située au-dessous du niveau de la mer. Ils vivent en villages. Il n'y a pas à proprement parler d'autorité politique coutumière. Chaque village a un roi de type sacré dont la fonction première est de réguler le cycle des pluies. Il doit également veiller à l'application des décisions prises par l'assemblée villageoise (katit) où tout adulte marié, homme ou femme, possède un droit de parole. L'organisation sociale repose sur trois unités sociologiques pertinentes: la division sexuelle des tâches; les classes d'âge et le culte aux puissances surhumaines appelées ukin (sg. bëkin).

4. J'utilise le manuel de Transcription des langues nationales (Dakar, République du Sénégal, avril 1972). Toutes les lettres se prononcent et en particulier : é comme "é » dans école ; ë comme « $œ u$ » dans cœur ; $g$, $s$ et $t$ toujours durs ; $j$ comme « di » dans diamant; $\tilde{n}$ comme "gn » dans agneau ; o comme dans étoffe ; ò comme dans eau ; u comme « ou » dans cou ; an comme «an » ou « en » dans enfant.

5. En ordre de grandeur, les religions de terroir représentent $51,3 \%$ de la population bissauguinéene (Michel CLÉVENOT (dir), L'état des religions dans le monde, Paris, La Découverte/Cerf, 1987, pp. 218-219). J'estime que cette proportion augmente à hauteur de 70 à $80 \%$ en pays Ajamat. Le catholicisme venu au XV $\mathrm{XV}^{\mathrm{e}}$ siècle avec les Portugais (René PÉLISSIER, Naissance de la Guinée. Portugais et Africains en Sénégambie (1841-1936), Orgeval, Pélissier, 1989), ne s'est réellement implanté dans le pays Ajamat qu'à la fin de la première moitié du $\mathrm{XX}^{\mathrm{e}}$ siècle. L'islam est relativement discret car l'économie locale était jusqu'à aujourd'hui (le régime marxiste est officiellement abandonné en 1983) peu propice aux commerçants Peuls, Mandings et Balantes.

6. Tous les Ajamats sont convaincus qu'une telle éventualité signifierait la destruction immédiate de la terre : elle exploserait pour les uns, elle serait engloutie par la mer pour les autres.

7. Le long de la frontière sénégalo-bissau-guinéene occupée également par des Ajamat, Émitay est appelé Ata Émit. Émit pourrait être la forme contractée de i-mir-ut = moi-savoir (saisir par la pensée)-pas. Ata est sans conteste le chef, l'auteur, celui à qui appartient, le responsable. Ay correspond à l'article défini au mais il a également le sens : « à la manière de ». Émitay comme Ata Émit pourraient dériver de Ata i mir ut ay: "à la manière d'un chef (de groupe) qu'on ne peut saisir en pensée ni dans son être ni par ses actes ». Cette étude linguistique a été réalisée en collaboration avec le père Jean-Pierre Assine (septembre 1994). 
8. Ésana avec près de 2000 habitants, est l'un des deux « gros bourgs » du pays Felup en GuinéeBissau : il est le lieu de notre travail depuis 1984.

9. En avril 1997, trois riziculteurs ont décomposé Émitay comme suit :

- é = particule interrogative pour interpeller une personne connue soit de nom soit parce qu'on a déjà eu à faire avec elle. L'interjection «oh?» ou " hé ?» est une bonne traduction. Le plus souvent, elle est employée dans un sens plus dur correspondant à l'exclamation « attends un peu, toi !» ou « un jour on se reverra!». Car elle rappelle que la première rencontre à été marquée par un acte fort : conflit, violence ou au contraire aide inattendue ;

- mit = radical indiquant l'action de reconnaître quelqu'un dont on va citer le nom. Une traduction approchante : «c'est bien toi x... ! ;

- ay = suffixe interrogatif : « de qui parle-t-on?».

Le suffixe ay annule tout espoir d'entendre le nom annoncé par le radical mit. Pour l'un de mes interlocuteurs, converti au catholicisme de longue date, la triple interrogation indique que seul Dieu peut être ainsi appelé.

10. Retenons parmi d'autres : Louis-Vincent THomAs, Les Diola. Essai d'analyse fonctionnelle sur une population de Basse-Casamance, Dakar, Mémoire de l'Institut Français d'Afrique Noire, 55, 1959, p. 587 ; ou encore, Nazaire UKËYËNG DIATTA, Anthropologie et herméneutique des rites joola (funérailles et initiation), thèse pour le Doctorat $3^{\mathrm{e}}$ cycle, Paris, EHESS, juin 1982 : p. 15, note 13.

11. Elle s'est déroulée en mai 1997, dans le campement installé dans la palmeraie de ce gardien d' ukin. Autour de nattes en feuilles de palmiste pour se reposer aux heures chaudes, le propriétaire entrepose son matériel de cueilleur de vin de palme, ses provisions d'eau et ses canaris de vin frais. Les hommes d'une même concession ou de même âge viennent boire et discuter des événements quotidiens à l'abri des oreilles villageoises.

12. Ce nom qui ne possède pas de singulier, désigne également le ciel.

13. Le Parti Africain pour l'Indépendance de la Guinée et du Cap Vert a été créé en 1956 (Paulette Pierson-Mathy, La naissance de l'État par la guerre de libération nationale: le cas de la Guinée-Bissau, Paris, UNESCO, 1980). La guerre de libération nationale débute en 1961-1962 et s'achève en 1974. Les Ajamat ont combattu dans les rangs de l'armée portugaise qui avait scellé une alliance par un sacrifice au bëkin du roi des rois de villages.

14. Bëkin (pl. ukin) est un terme polysémique qui désigne à la fois une puissance surhumaine, le sanctuaire, l'autel et le mobilier du culte. Il en existe plusieurs centaines par village. On peut distinguer entre les ukin qui servent les intérêts de la communauté villageoise (royauté, maternité, initiation masculine, divination, activité de guerre ou de chasse, etc.) de ceux qui se rapportent aux intérêts du lignage (ancêtre, rizière, la forge, thérapie). Intercesseur auprès de Émitay, ils sont garants de l'ordre social parce qu'ils sont des repères au sol des lois divines. Chaque bëkin requiert un desservant (kauasen aku, "celui qui verse ») et selon son importance, des adjoints et des affiliés. Rêve, maladie élective et initiation constituent la voie d'accès à cette fonction.

15. En raison de la raréfaction des pluies, l'époque des labours est de plus en plus réduite. Le travail au kayendo, sorte de pelle en forme de rame et à long manche, se poursuit alors tard dans la nuit.

16. Sans entrer dans un long développement, il faut noter que la confluence du Rio Cacheu et de la mer constitue un lieu hautement symbolique. En effet, c'est dans l'océan Atlantique que résident ordinairement les ukin tandis que les doubles de l'homme habitent les marigots du delta du fleuve (hippopotame, marsouin, lamentin).

17. Ceiba pentandra (L.) Gaertn, plus couramment appelé fromager buyogumabu).

18. C'est pourquoi la langue Felup qui les place toujours en début de phrase, dispose d'un choix extraordinaire de substantifs précis et concrets. Il y a, par exemple, six noms pour désigner le riz : en tant que plante, pas encore pilé, pilé en quantité, bouilli, cuit et pas cuit. Je remercie le père Guiseppe Fumagali qui m'a autorisé à prendre copie de son étude inédite: Appulti di 
grammatica della lingua Felupe parlata a Suzana, Missao Catolica, Suzana, manuscrit dactylographié, mars 1977, p. 9.

19. Commun en Afrique noire, le soufflet de forge est un tronc d'arbre d'une longueur moyenne de $80 \mathrm{~cm}$, évidé en forme de poire : la partie effilée sert de tuyau. Sur l'autre extrémité s'ouvrent verticalement deux cheminées séparées et coiffées hermétiquement d'une peau de chèvre. Le client actionnera ces deux peaux à la manière de deux pistons pendant que le forgeron travaillera l'outil qu'il lui a commandé.

20. Il faut noter que les hommes se sont montrés plus réservés sur la corrélation entre tornade et courroux divin. Par ailleurs, les femmes qui m'ont parlé dans ce sens, appartiennent très souvent à la classe d'âge des 40 ans et travaillent une grande partie de l'année dans une ville soit du Sénégal soit à Bissau même.

21. R. CoLIN, Littérature africaine d'hier et de demain, Paris, ADEC, 1965, cité par Louis-Vincent THомAS, « Afrique noire. Littérature », in Encyclopœdia Universalis, Paris, 1994.

22. Dans mes travaux précédents, je n'avais pas attribué cette place à asimau: en collaboration avec Odile JOURNET, «L'indistinction du corps. Savoirs, thérapies et religion chez les Joola-Felup (Guinée-Bissau)», in Marie-Josée JoLIVET et Diana REY-HuLMAN (dir), Jeux d'identités. Études comparatives à partir de la Caraibe, Paris, L'Harmattan, 1993, pp. 311-336. Ses liens étroits avec le développement de la personnalité, n'étaient pas apparus aussi nettement qu'aujourd'hui dans les entretiens. Une familiarité réciproque a levé partiellement les réticences à parler du sang.

23. Citation publiée dans Odile JOURNET et André JULLIARD, art. cit., 1993, p. 324, auquel je me permets de renvoyer pour une approche plus complète de l'anthropologie physique Ajamat.

24. Un autre indice de ce lien avec le geste ordinaire par lequel on clôture toute parole dont on veut souligner la radicalité des propos. La main en porte-voix balaie la bouche d'un mouvement latéral en chuintement l'air soufflé : littéralement, on coupe le souffle.

25. L'intervention de Émitay se limite le plus souvent «à faire diminuer » la quantité de sang chez l'homme qui doit mourir par sa volonté. Il empêche la régénération du liquide sanguin aussi bien chez le blessé mortel que chez le vieillard qui à fait son temps sur terre.

26. Le corps chaud est un symptôme qui entre dans le tableau des critères nosographiques des maladies tant au Sénégal qu'en Guinée-Bissau. Il est très difficile à caractériser. Une patiente rencontrée dans le dispensaire d'État, le décrit comme une inflammation fiévreuse de la face inférieure de l'épiderme qui se met à transpirer.

27. Il n'y a pas contradiction avec le fait que le cœur ne soit pas une pièce maîtresse du système sanguin. À Ésana, chacun sait par l'observation routinière du dépeçage du gibier ou des animaux sacrifiés qu'ils sont dans un rapport de circulation.

28. Il y a au moins « un souffle animal » qui peut tuer : celui des doubles. Par exemple, lorsqu'un chasseur abat un hippopotame, il doit immédiatement se mettre derrière sa tête pour se protéger du souffle expiré. Le recevoir sur soi, c'est la mort assurée.

29. De l'avis général, « le cœur n'a pas beaucoup de maladies [car] il est protégé par Émitay». Il n'est pas sujet à des transformations morphologiques comme les autres organes auxquels il est associé. 


\section{RÉSUMÉS}

La religion de terroir chez les Diola Adiamat (nord-ouest de la Guinée-Bissau) associe l'idée d'un Dieu unique et créateur (Emitay), et une activité religieuse (sacrifice, libation) à caractère polythéiste représentée par un ensemble de puissances surhumaines (ukin) qui régule le social. Emitay est un dieu distant - position appelée à se modifier par la confrontation récente avec l'islam et surtout le catholicisme - qui manifeste cependant une présence agissante sans pour autant prendre la forme d'un culte. Le vent qu'Il produit, est l'un des moyens d'expression par lequel Dieu engage au moins deux relations directes avec les hommes. L'une personnelle car en posant l'appareil respiratoire comme axe biologique du corps humain, Il anime constamment la respiration de l'individu. À chacun, ensuite, de veiller à la régularité du système en évitant toutes « surpressions d'aire " dues à l'exacerbation des sentiments dans les relations à autrui (colère, peur, jalousie). L'autre collective car Emitay envoie des épidémies par le vent pour marquer son courroux contre l'accroissement des morts par «sorcellerie ». À côté des sacrifices nécessaires pour remédier aux malheurs biologiques individuels ou collectifs, seule la parole des hommes qui explique l'événement et, à travers lui, la Loi divine, constitue la véritable attitude sociale et religieuse face à Dieu.

The local religion of the Diola Adiamat people (who live in the Northwest of Guinea-Bissau) associates the idea of single God creator of all thins (Emitay) and religious activities (sacrifices, libations) whose polytheistic nature is represented by a group of superhuman powers (ukin) which regulate the social order. Emitay is a distant God - a position which is undergoing some change given the recent confrontation with Islam and, more importantly, with Catholicism who, nevertheless, has an active presence though it is not embodied in a cult. The wind He produces is one of the means of expression through which God creates at least two direct relationships with men. One is personal, since by positing the respiratory apparatus as a biological axis of the human body, He constantly animates the individual's respiration. It is then up to each individual to take care of the regularity of the System by avoiding the overpressure of air caused by the exacerbation of feelings toward other people (anger, fear, jealousy). The other relation is collective since through the wind Emitay sends epidemies in order to indicate His wrath caused by the increasing number of deaths through "witcheraft". Besides the sacrifices necessary to remedy the individual or collective biological mishaps, the human discourse which explains the events and, through them, the Divine Law, represents the only possible social and religious attitude toward God.

La religión del terruño entre los Diola Adiamat (nor-oeste de Guinea) asocia la idea de un Dios único y creador (Emitay) con una actividad religiosa (sacrificio, libación) de tipo politeista, representada por un conjunto de potencias sobrehumanas (ukin) que regulan lo social. Emitay es un Dios distante (postura que esta cambiando por la confrontación reciente con el Islam y, sobre todo, con el catolicismo), que manifiesta sin embargo una presencia activa, la cual no toma por lo tanto la forma de un culto. El viento que El produce es uno de los medios de expresión a través del cual Dios tiene por lo menos dos relaciones directas con los hombres. La primera es de tipo personal, pues si se considera que el sistema respiratorio es el eje biológico del cuerpo humano, significa que El anima de manera constante la respiración del individuo. Lueglo toda "falta de 
aire" que podría surgir por exacerbación de los sentimientos en las relaciones al Otro (furor, miedo, celosía). La segunda relación es de tipo colectivo, porque Emitay manda epidemias a través del viento, para dar a conocer su ira contra el crecimiento de los fallecimientos por "brujería". En este contexto, y al lado de los sacrificios necesarios para remediar las desgracias biológicas, individuales o colectivas, la palabra humana que explica el acontecimiento y, a través de éste, la Ley divina, se constituye como la única y verdadera postura social y religiosa frente a Dios.

\section{AUTEUR}

ANDRÉ JULLIARD

CNRS - Université Blaise Pascal - Clermont II 